\title{
Erratum to: An Up-regulation of IRF-1 After a Spinal Cord Injury: Implications for Neuronal Apoptosis
}

\author{
Jian Zhao ${ }^{1}$ Chen Chen ${ }^{2} \cdot$ Jian-Ru Xiao $^{1} \cdot$ Hai-Feng Wei ${ }^{1} \cdot$ Xu-hui Zhou ${ }^{1} \cdot$ Xing-Xing Mao ${ }^{2}$. \\ Wei-dong Zhang ${ }^{2} \cdot$ Rong Qian ${ }^{2} \cdot$ Xin-lei Chen ${ }^{2} \cdot$ Ming-qing $\mathrm{He}^{4} \cdot$ Xiao-Wei Yu ${ }^{3,5} \cdot$ Jian Zhao ${ }^{1}$
}

Published online: 21 March 2016

(C) Springer Science+Business Media New York 2016

\section{Erratum to: J Mol Neurosci (2015) 57:595-604 DOI 10.1007/s12031-015-0642-2}

The original version of this article unfortunately contained a missing reference information. This should be cited in the text, as presented below:

Seung-Won Park et al., JPET 320:1001-1012, 2007 (cited below) showed that IRF-1 mRNA is up-regulated in spinal cord injury in rats, and this increase is blocked by thiozolidinedione derivatives.

\section{References}

Park S-W, Yi J-H, Miranpuri G, Satriotomo I, Bowen K, Resnick DK, Vemuganti R (2007) Thiazolidinedione class of peroxisome proliferator-activated receptor $\gamma$ agonists prevents neuronal damage, motor dysfunction, myelin loss, neuropathic pain, and inflammation after spinal cord injury in adult rats. JPET 320:1001-1012

The online version of the original article can be found at http://dx.doi.org/ 10.1007/s12031-015-0642-2.

\author{
Xiao-Wei Yu \\ njh306@163.com \\ drzhaojian123@163.com
}

$\triangle$ Jian Zhao

1 Department of Orthopedics, Shanghai Changzheng Hospital, Shanghai 200000, China

2 Department of Orthopedics, Affiliated Hospital of Nantong University, Nantong, Jiangsu Province 226001, China

3 Department of Neurology, Affiliated Hospital of Nantong University Nantong Second People's Hospital, Nantong, Jiangsu Province 226001, China

4 Department of Geriatrics, Affiliated Hospital of Nantong University, Nantong, Jiangsu Province 226001, China

5 Department of Neurology, Affiliated Hospital of Nantong University Nantong Second People's Hospital, Nantong, Jiangsu Province 226001, China 